\title{
A Hybrid 1D and 3D Approach to Hemodynamics Modelling for a Patient-Specific Cerebral Vasculature and Aneurysm
}

\author{
Harvey Ho ${ }^{1}$, Gregory Sands ${ }^{1}$, Holger Schmid ${ }^{2}$, Kumar Mithraratne ${ }^{1}$, \\ Gordon Mallinson ${ }^{3}$, and Peter Hunter ${ }^{1}$ \\ 1 Bioengineering Institute, University of Auckland, New Zealand \\ \{harvey.ho,g.sands, p.mithraratne, p.hunter\}@auckland.ac.nz \\ 2 Department of Continuum Mechanics, RWTH Aachen University, Germany \\ schmid@km.rwth-aachen.de \\ 3 Department of Mechanical Engineering, University of Auckland, New Zealand \\ g.mallinson@auckland.ac.nz
}

\begin{abstract}
In this paper we present a hybrid 1D/3D approach to haemodynamics modelling in a patient-specific cerebral vasculature and aneurysm. The geometric model is constructed from a 3D CTA image. A reduced form of the governing equations for blood flow is coupled with an empirical wall equation and applied to the arterial tree. The equation system is solved using a MacCormack finite difference scheme and the results are used as the boundary conditions for a $3 \mathrm{D}$ flow solver. The computed wall shear stress (WSS) agrees with published data.
\end{abstract}

\section{Introduction}

Intracranial aneurysms are dilated arterial lesions in the brain. The majority of them are saccular shaped and arise from the Circle of Willis $(\mathrm{CoW})$, the ring of vessels formed at the skull base (Fig. 1a). When a cerebral aneurysm ruptures, blood will flow into the subarachnoid space causing subarachnoid hemorrhage (SAH), which has a high mortality and morbidity rate [1]. Although the mechanisms underlying the formation of aneurysms are still not fully understood, it has been suggested that haemodynamic factors play an important role in their genesis and development [2].

To better understand these factors, many research groups have studied the flow patterns in cerebral aneurysms e.g. in [2|3|4|5]. The techniques used include non-invasive in vivo MR imaging [3, in vitro phantom experiments [4, and three-dimensional (3D) computational fluid dynamics (CFD) modelling [2], or a combination of these techniques [5]. Among these methods, well validated CFD has the advantage of being able to predict flow at locations where in-vivo flow data are difficult to obtain. The problem of 3D CFD models, however, is that a huge number of computational elements are generally required to capture complex flow patterns in tortuous vessels and bifurcations, therefore making 3D modelling for large vasculatures computationally infeasible. On the other hand, 


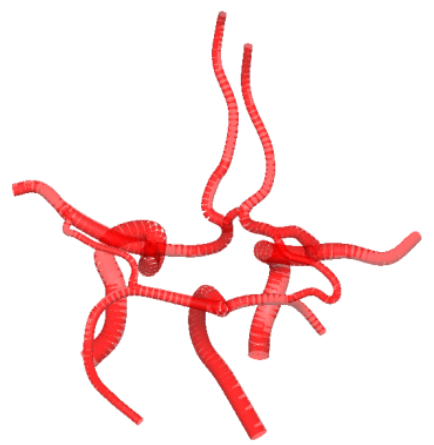

(a)

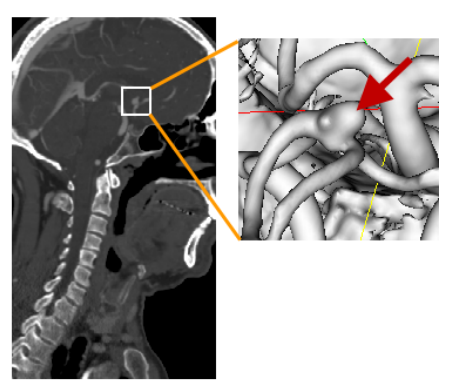

(b)

Fig. 1. (a) Circle of Willis (posterior view); (b) A CTA image: the arrow indicates an aneurysm at the anterior communicating artery (ACoA)

blood flow in a vasculature can be modelled with reduced 1D formulations of the governing equations, as used to model flow in cerebral and coronary arterial trees e.g. in 667. However, the 1D models are not capable of capturing complex flow patterns, such as vortices, flow separation and reattachment, or flow reversal as in 3D models.

To absorb the strength from both $1 \mathrm{D}$ and 3D modelling strategies, some research groups have investigated hybrid or multidimensional modelling strategies [89]. The philosophy is to employ a 3D CFD model to analyze the flow in the vessel of interest in high detail, and use the 1D CFD model for the remaining part of the arterial tree. The result is a reduced number of parameters and substantially decreased computational cost [9].

This work takes a similar approach i.e. by applying a hybrid 1D/3D modelling technique to haemodynamic analysis in a patient-specific cerebral aneurysm, which grows at the anterior communication artery (ACoA) (Fig. 1b). The difference between our work and that of [89] is that both the 1D arterial tree and the $3 \mathrm{D}$ aneurysm in our model are digitized from a 3D CTA image, and therefore reflect the actual vascular anatomy.

\section{Method}

\subsection{Vascular Model Construction}

Arterial Tree. The 3D CT Angiography (CTA) image of Fig. 1(b) contains 421 slices of $378 \times 336$ pixels. The spatial resolution of the image is $0.488 \times$ $0.488 \times 0.7 \mathrm{~mm}$. Using the open source imaging and visualization tool CMGUI we manually select 175 key points along the centre line of large blood vessels as nodes. The radius at each node is defined as a field for that particular node. These nodes are then connected by 1D cubic Hermite elements. Cylinders are constructed along these elements to represent the major arteries supplying blood to the brain. Fig. 2 depicts this digitization process. 


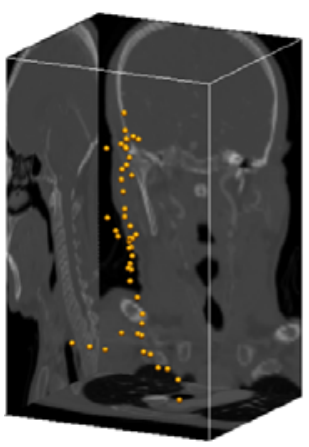

(a)

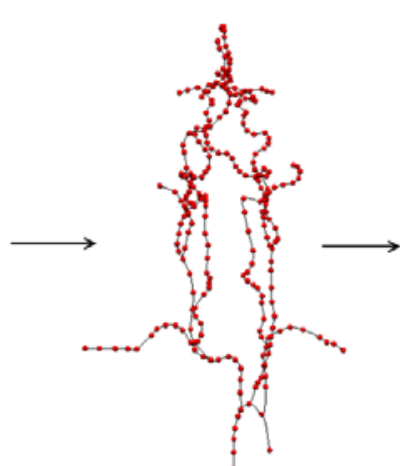

(b)

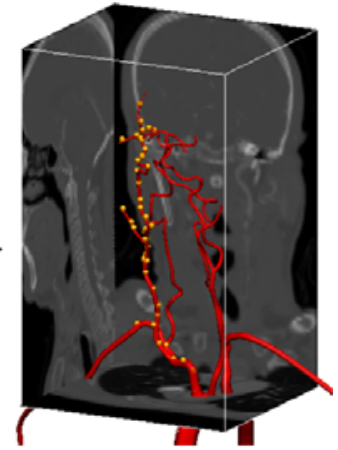

(c)

Fig. 2. Vascular tree construction pipeline: (a) Node selection; (b) 1D elements construction; (c) Cylinder simulation incorporating radius information

Aneurysm Models. Using CMGUI, the isosurface of the aneurysm lumen is extracted from the volume image as an isovalue of the image intensity at the lumen boundary. The initial isosurface (triangular mesh) is further repaired by mesh smoothing or decimation algorithms, which are available in CMGUI. Fig. $3(\mathrm{a})$ and 3(b) show the initial triangular mesh extracted from the volume image, and the surface mesh after repairing.

Computational Grid Generation. As with other Finite Element Analysis software, the geometrical model must be split into a finite set of elements, the so-called computational grid, to be used for flow analysis. In this work we employ a commercial grid generator ANSYS ICEM and use the octree method, which results in the grid shown in Fig. 3(c).

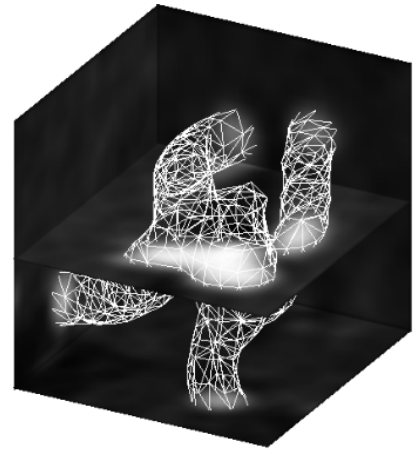

(a)

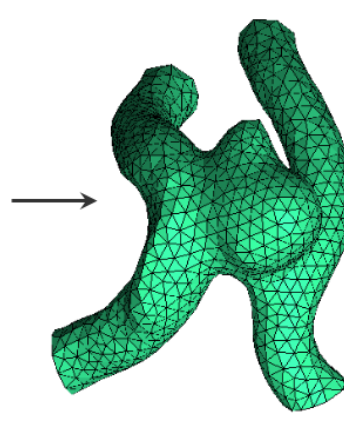

(b)

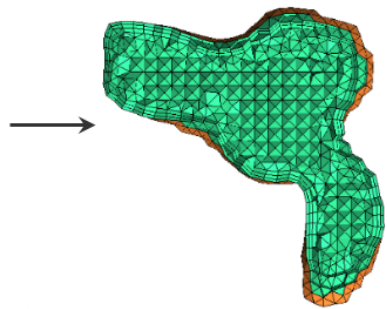

(c)

Fig. 3. (a) Isosurface extracted from volume image; (b) Surface triangular mesh after repairing; (c) Computational grid at the cross-section of aneurysm 


\subsection{Haemodynamics Modelling}

1D Model. In large arteries, the relative size of red blood cell to vessel diameter is small and blood can be modeled as an incompressible, homogeneous, Newtonian fluid 6 6/7/8. If we further assume that the flow in the circumferential direction is negligible and that the radial velocity is small compared to axial velocity, then the governing equations can be reduced to two equations. By further including a constitutive wall equation [7] we get:

$$
\begin{gathered}
\frac{\partial R}{\partial t}+V \frac{\partial R}{\partial x}+\frac{R}{2} \frac{\partial V}{\partial x}=0 \\
\frac{\partial V}{\partial t}+(2 \alpha-1) V \frac{\partial V}{\partial x}+2(\alpha-1) \frac{V^{2}}{R} \frac{\partial R}{\partial x}+\frac{1}{\rho} \frac{\partial p}{\partial x}=-2 \frac{v \alpha}{\alpha-1} \frac{V}{R^{2}} \\
p(R)=G_{o}\left[\left(\frac{R}{R_{o}}\right)^{\beta}-1\right]
\end{gathered}
$$

where $P, R, V, \rho$ and $v$ represent pressure, inner vessel radius, velocity, blood density and viscosity respectively. The parameter $\alpha$ specifies axial velocity profile. $G_{o}$ is a stiffness reference, $R_{o}$ is the unstressed radius, and $\beta$ is the wall elasticity coefficient. The hyperbolic set of nonlinear partial differential equations (1-3) is solved numerically using a second order MacCormack finite difference method. Furthermore, a bifurcation model is incorporated to predict flow distribution, velocity and pressure gradient across branches, and thus the whole arterial tree [7.

3D Model. The full 3D version of the governing Navier-Stokes equation can be expressed in a vector form:

$$
\begin{aligned}
\nabla \cdot \mathbf{v} & =0 \\
\rho\left(\frac{\partial \mathbf{v}}{\partial \mathbf{t}}+\mathbf{v} \cdot \nabla \mathbf{v}\right) & =-\nabla p+\nabla \cdot \tau
\end{aligned}
$$

where $\mathbf{v}$ represents the flow velocity in 3D. The practice of 3D CFD modelling is to discretize the equations (445) over the computational mesh of the physical domain (in this case the cerebral aneurysm), and solve the system numerically. The flow solver employed in this work is a finite volume based CFD solver, ANSYS CFX [10], which has a well defined interface with the grid generator ICEM. In brief, the process of patient specific CFD modelling can be streamlined into a pipeline:

(a) the surface mesh of a vascular structure is extracted from medical images (e.g. by using a threshold isovalue, or other image processing methods);

(b) the initial surface mesh is repaired, improved and translated into a format (e.g. the stereolithography format STL) acceptable to a grid generator;

(c) a computational grid is generated (e.g. by using ICEM) and exported to a 3D flow solver (e.g. CFX-Pre); 
(d) the flow and domain configuration, the initial and boundary conditions plus solver parameters are specified (e.g. in CFX-Pre);

(e) the (transient) flow is solved (e.g. by using CFX);

(f) the results are post-processed (e.g. in CFX-Post or CMGUI).

Steps (a)-(c) in the pipeline constitute the pre-processing block, (d)-(e) the solver block and (f) the post-processing block.

Coupling of 1D/3D Solvers. The 1D and 3D solvers are coupled in step (d) of the pipeline. The flow solver CFX allows transient waveforms to be prescribed as boundary conditions and these waveforms must be expressed as functions of time. The waveforms calculated from the $1 \mathrm{D}$ model, however, are not analytical functions but discrete numeric values. Hence, we perform Fourier analysis for these waveforms and supply their leading ten harmonics to CFX.

\section{Results}

1D Model. When solving the governing equations, the density $\rho$ and viscosity $\nu$ of the blood are set as $1.05 \mathrm{~g} / \mathrm{cm}^{3}$ and $3.2 \mathrm{~cm}^{2} / \mathrm{s}$ respectively. The initial velocity and pressure at all vessel segments are $0 \mathrm{~mm} / \mathrm{s}$ and $10.6 \mathrm{kPa}(80 \mathrm{mmHg})$ respectively. The spatial and temporal step of the finite difference grid is set as $1 \mathrm{~mm}$ and 0.1 millisecond, respectively. A physiological pulsatile pressure $(80 \mathrm{mmHg}-$ $120 \mathrm{mmHg}$ ) is prescribed from the inlet i.e. the ascending aorta. The pressure at outlets is fixed at $80 \mathrm{mmHg}$. The pressure gradient between the inlet and outlets therefore drives blood flow through the arterial tree. It takes about three minutes to compute the flow during a cardiac cycle using a laptop PC (1.73 $\mathrm{GHz}$, Intel Pentium Dual-Core) and the resulting pressure distribution at four distinct phases of a cardiac cycle is shown in Fig. 4a. The velocity profiles at three locations of the aneurysm region are shown in Fig. 4b and the data from sites $\mathbf{B}$ and $\mathbf{C}$ will be passed to the $3 \mathrm{D}$ model as boundary conditions.

3D Model. The computational grid of Fig. 3c contains about 129,500 elements, which have a combination of tetrahedra and pentahedra. The boundary conditions are set as follows: the velocities computed from the $1 \mathrm{D}$ model (at sites $\mathbf{B}$ and $\mathbf{C}$ of Fig. 4b) are used as the inflow boundary conditions for the 3D model. The zero pressure boundary condition is prescribed from outlets. The viscoelastic properties of vessel wall are ignored and the no-slip wall boundary condition is applied. The flow type is defined as laminar which is justified by the highest Reynolds number (754) which occurs at the ventricular ejection phase. The computational results include important flow data such as pressure, velocity in the whole fluid domain and wall shear stress (WSS) at the vessel wall. The distribution of WSS at time steps $0.15 \mathrm{~s}, 0.3 \mathrm{~s}, 0.5 \mathrm{~s}$ and $0.9 \mathrm{~s}$ are visualized in Fig. 5 h. These time steps represent four different phases of cardiac cycle as illustrated in the pressure profile chart of Fig. 5. The streamlines in Fig. 5b which bears the flow velocity information visualize the pathway of blood flow. 


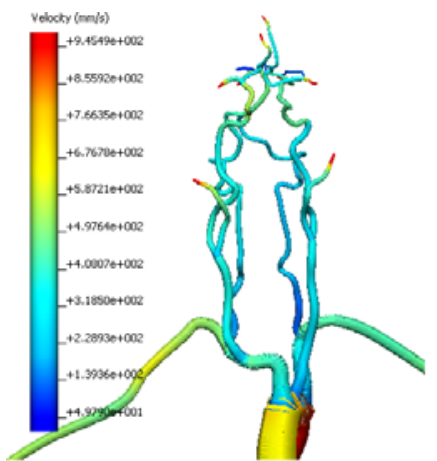

(a)
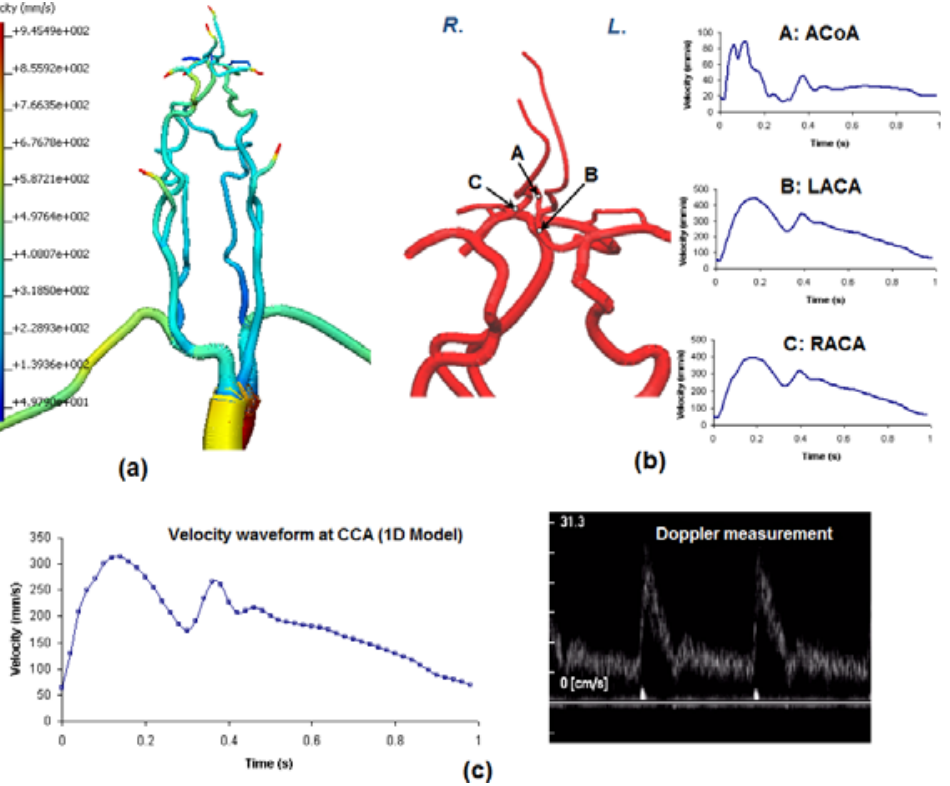

(c)

Fig. 4. Haemodynamics modelling in the cerebral vasculature: (a) Velocity distribution in the arterial tree at $0.2 \mathrm{~s}$; (b) A closer look at cerebral arteries: A - the communicating artery where the aneurysm grows; B,C - two inlets on ACA; (c) Velocity waveform at CCA: Left-1D model; Right-Doppler measurement

Model Validation. A LogicScan 128 ultrasound scanner (TELEMED Ltd., Lithuania) is used to detect the flow velocity at the inner carotid artery to validate the $1 \mathrm{D}$ result. The measured waveform, which varies between $55 \mathrm{~mm} / \mathrm{s}$ to $310 \mathrm{~mm} / \mathrm{s}$ during a cardiac cycle, is shown in Fig. 4k. A comparison with the 1D model indicates that the largest velocity (about $31-32 \mathrm{~cm} / \mathrm{s}$, occurs at systole) of the simulation matches that of the ultrasonic data. However, our model overestimates the flow velocity at diastole. Overall, we consider the simulation result is within the acceptable physiological range of in vivo measurement.

We also compare the computed WSS data with the results published by other research groups e.g. in [25]. The comparison is tabulated in Table 1 and it shows that our result agree favourably with the published data.

\section{Discussion}

A number of in vivo, in vitro measurements and CFD modellings (e.g. in [2345]) have been performed to study the WSS induced by blood flow and its relationship with aneurysm genesis. The problem with the CFD approach is that a huge computational cost is required for full 3D analysis of a large vasculature. In this work we adopt a hybrid 1D/3D approach to a patient-specific cerebral vasculature and aneurysm. The benefits of such a strategy are obvious: (1) the difficulties arising 


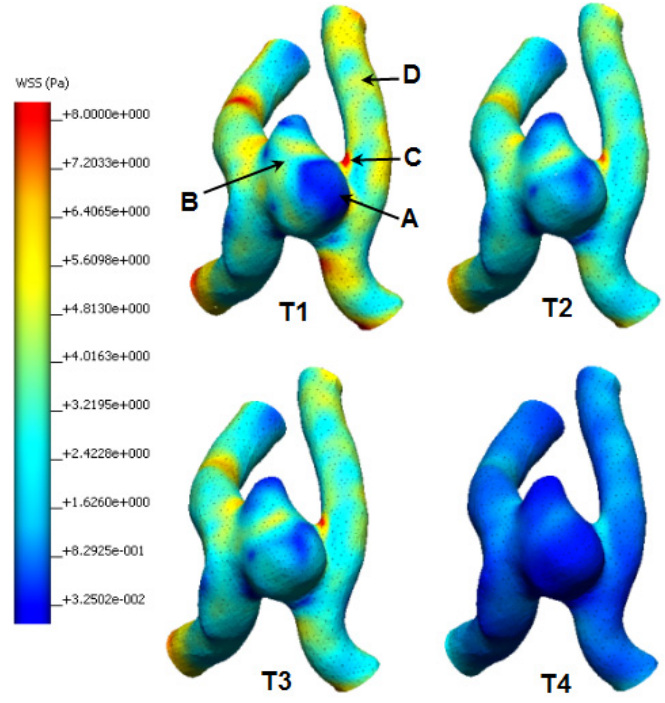

(a)
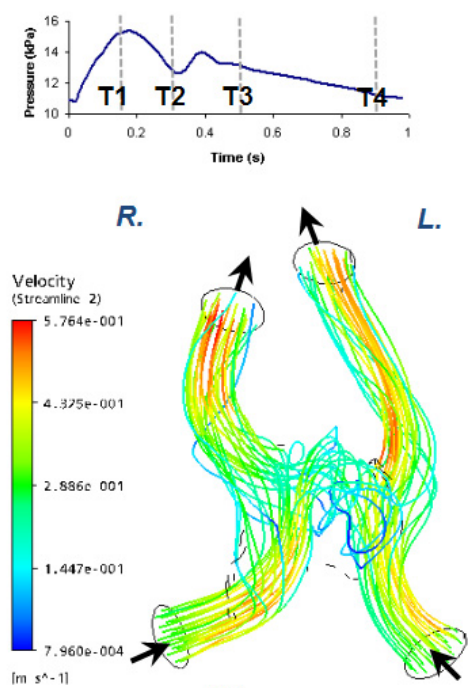

(b)

Fig. 5. Postprocessing of data for the aneurysm (posterior view). (a) WSS on vessel wall at 4 time steps, postprocessed in CMGUI; (b) Streamline in the fluid domain, postprocessed in CFX-Post.

Table 1. Comparison of flow data in literatures

\begin{tabular}{c|c|c|c|c|c|c|c|c|c}
\hline Time step & \multicolumn{2}{|c|}{ Our model at selected sites } & \multicolumn{2}{|c|}{ Shojima et al [2] } & \multicolumn{2}{|c}{ Steinman et al [5] } \\
\hline & A(Dome) & B & C(Neck) & D & Dome & Sac & Neck & Dome & Sac \\
\hline T1 & 0.35 & 2.65 & 9.87 & 5.73 & - & - & - & - & - \\
T2 & 0.86 & 2.77 & 6.52 & 4.03 & - & - & - & - & - \\
T3 & 0.71 & 2.78 & 6.98 & 4.34 & - & - & - & - & - \\
T4 & 0.24 & 0.55 & 1.68 & 0.84 & - & - & - & - & - \\
\hline Average & 0.54 & 2.18 & 6.26 & 7.52 & $0.3-0.5$ & $1-5$ & $8-10$ & $0.4-0.8$ & $1.6-2$ \\
\hline
\end{tabular}

from the treatment of boundary conditions for 3D models are handled naturally from the 1D model; and (2) the computational cost is substantially reduced [9]. At regions of interest, the $3 \mathrm{D}$ model reveals more flow information (e.g. the streamline in Fig. 5b) which cannot be captured by a pure 1D model.

It is worth noting that in this work we assumed that the arterial wall is elastic in the $1 \mathrm{D}$ model but rigid in the $3 \mathrm{D}$ model. That is to say, we ignored the wall deformation during a cardiac cycle in $3 \mathrm{D}$ modelling. This is an acceptable approximation for intracranial arteries because they are stiffer than extracranial arteries 56. However, the same assumption may not hold true for carotid arteries and certainly not for the aorta. In the latter case, a more complex deformable wall model and an Arbitrary Lagrangian Eulerian (ALE) formation of the governing equations need to be solved, and this remains as our future work. 


\section{Conclusion}

In this study we used a hybrid $1 \mathrm{D} / 3 \mathrm{D}$ method to model a patient-specific aneurysm and the surrounding vasculature. We have developed a computational pipeline which starts from vascular model construction, to grid generation, and to $1 \mathrm{D} / 3 \mathrm{D}$ CFD modelling. The pipeline leads to a substantial reduction of computational cost. The initial results agree with previously published data. Future work include applying such a pipeline to CFD analysis of aneurysms arising from other (intracranial) arteries.

\section{References}

1. Suarez, J.I., Tarr, R.W., Selman, W.R.: Aneurysmal subarachnoid hemorrhage. The New England Journal of Medicine 354(4), 387-396 (2006)

2. Shojima, M., Oshima, M., Takagi, K., Torii, R., Hayakawa, M., Katada, K., Morita, A., Kirino, T.: Magnitude and role of wall shear stress on cerebral aneurysm. Stroke 35, 2500-2505 (2004)

3. Cebral, J., Yim, P.J., Lohner, R., Soto, O., Choyke, P.L.: Blood flow modeling in carotid arteries with computational fluid dynamics and MR imaging. Academic Radiology 9(11), 1286-1299 (2002)

4. Liou, T., Li, Y., Juan, W.: Numerical and experimental studies on pulsatile flow in aneurysms arising laterally from a curved parent vessel at various angles. Journal of Biomechanics 40, 1268-1275 (2007)

5. Steinman, D., Milner, J., Norley, C.J., Lownie, S.P., Holdsworth, D.W.: Imagebased computational simulation of flow dynamics in a giant intracranial aneurysm. American Journal of Neuroradiology, 559-566 (2003)

6. Alastruey, J., Parker, K., Peiro, J., Byrd, S., Sherwin, S.: Modelling the circle of willis to assess the effects of anatomical variations and occlusions on cerebral flows. Journal of Biomechanics 40, 1794-1805 (2007)

7. Smith, N.P., Pullan, A.J., Hunter, P.J.: An anatomically based model of transient coronary blood flow in the heart. SIAM Journal of Applied Mathematics 62(3), 990-1018 (2000)

8. Formaggia, L., Gerbeau, J.F., Nobile, F., Quarteroni, A.: On the coupling of 3d and $1 \mathrm{~d}$ navier-stokes equations for flow problems in compliant vessels. Computer Methods in Applied Mechanics and Engineering 191, 561-582 (2001)

9. Urquiza, S.A., Blanco, P.J., Venere, M.J., Feijoo, R.: Multidimensional modelling for the carotid artery blood flow. Computer Methods in Applied Mechanics and Engineering 195, 4002-4017 (2006)

10. ANSYS: ANSYS CFX-Solver, Release 10.0: Theory. ANSYS Europe Ltd (2005) 